\title{
The physiological cost of male-biased parasitism in a nearly monomorphic mammal
}

\author{
Arturo Oliver-Guimerá1,2, Carlos Martínez-Carrasco ${ }^{1}$, Asta Tvarijonaviciute ${ }^{3}$, María Rocío Ruiz de Ybáñez², \\ Jordi Martínez-Guijosa ${ }^{4}$, Jorge Ramón López-Olvera', Xavier Fernández-Aguilar ${ }^{1}$, Andreu Colom-Cadena', \\ Gregorio Mentaberre ${ }^{1}$, Roser Velarde ${ }^{1}$, Diana Gassó ${ }^{1}$, Mathieu Garel ${ }^{5}$, Luca Rossi ${ }^{6}$, Santiago Lavín ${ }^{1}$ \\ and Emmanuel Serrano ${ }^{1,7^{*}}$ (D)
}

\begin{abstract}
Background: Even though male-biased parasitism is common in mammals, little effort has been made to evaluate whether higher parasitic burden in males results in an extra biological cost, and thus a decrease in fitness. Body condition impairment and the augmentation of oxidative stress can be used as indicators of the cost of parasite infections. Here, we examined relationships between gastrointestinal and respiratory helminths, body condition and oxidative stress markers (glutathione peroxidase, paraoxonase-1) in 28 Pyrenean chamois (Rupicapra p. pyrenaica) sampled in autumn.
\end{abstract}

Results: Only male chamois showed a reduction in body condition and higher oxidative stress due to parasite infection, likely because of the extremely high parasite burdens observed in males.

Conclusions: This study made evident a disparity in the physiological cost of multiple parasitism between sexes in a wild mammal, mainly due to parasitic richness. Because of the similar life expectancy in male and female chamois, we suggest that males may have developed natural mechanisms to compensate for higher parasite loads during the rut.

Keywords: Gastrointestinal nematodes, Lung nematodes, Kidney fat reserves, Oxidant/antioxidant status, Rupicapra pyrenaica pyrenaica

\section{Background}

Sex-based differences in exposure, susceptibility [1] and tolerance lead to differences in parasite prevalence [2], intensity [3], and pathology [4] in a broad range of vertebrate species. Males are often more infected than females as observed in birds [5], rodents, bats [6], ungulates [1,3] and humans [4]. This male-biased parasitism has been linked to both hormonal and behavioural differences. Generally, males invest more energy than females in the development of testosterone-mediated

\footnotetext{
* Correspondence: emmanuel.serrano.ferron@gmail.com

${ }^{1}$ Servei d'Ecopatologia de Fauna Salvatge (SEFaS), Wildlife Health Service, Departament de Medicina i Cirurgia Animal, Universitat Autònoma de Barcelona, E-08193 Bellaterra, Spain

${ }^{7}$ Departamento de Biologia \& CESAM, Universidade de Aveiro, 3810-193

Aveiro, Portugal

Full list of author information is available at the end of the article
}

traits, such as secondary sexual traits or courtship displays. Although testosterone is necessary for developing of secondary sex characteristics in males, high testosterone levels have been linked to a suppressed immune system $[4,7]$ and increased parasitism. Malebiased infection is common in sexually dimorphic and polygynous species [8], and might reduce male fitness. However, in sexually dimorphic species it might be difficult to separate the physiological costs of parasitism from the costs of the production and maintenance of sexually-selected traits [9]. Monomorphic species, in which males and females are morphologically similar, provide ideal systems for examining the physiological costs of male-biased parasitism.

Here we compare the physiological costs of malebiased parasitism in a nearly monomorphic ungulate 
(only small differences in body mass can be observed in autumn), the Pyrenean chamois (Rupicapra pyrenaica pyrenaica). Found in the mountains of southern Europe, chamois are medium-sized polygynous ungulates [10]. Males are more heavily parasitized [3], however, life expectancy is similar between the sexes [10].

We assess the physiological costs of infection using both general indicators of nutritional status, such as indicators of body condition (e.g. kidney fat stores, KFs [11]) and insulin-like growth factor 1 (IGF1), and oxidative stress (OS) markers, such as paraoxonase 1 (PON1), total antioxidant capacity (TAC) and glutathione peroxidase (GPX). Oxidative stress originates from an imbalance between the production of reactive oxygen or nitrogen species, and the capacity of the organism to compensate for their detrimental effects or destroy these harmful products [12]. As result, OS biomarkers are widely used to measure infection damage in veterinary sciences [13] and conservation physiology [14] and are considered good animal health indicators of animal health. The proper assessment of oxidative status must consider not only the concentration of antioxidants (e.g. antioxidant vitamins or endogenous enzymes), but also oxidative damage. For example, an elevation in endogenous antioxidants with stable oxidative damage concentrations suggests that individuals are successfully dealing with oxidative stress. In contrast, the depletion of antioxidant substances and stable oxidative conditions suggest oxidative damage.

In this work, we assess the endoparasite (gut and respiratory helminths) and physiological status (oxidative stress and nutritional status) of 28 adult Pyrenean chamois. Using these data, we tested whether, for a given parasite load, males have a higher physiological cost than females. Because male chamois are prone to suffer from malnourishment due to rut-induced hypophagia (mainly territorial individuals, see [15]), we expect higher physiological costs (i.e. lower nutritional status and higher oxidative stress) in males than in females for a given parasite burden. On the other hand, because both female and male chamois have similar life expectancies we expect that males may have developed physiological adaptations to compensate for the damage caused by parasites during the rutting period.

\section{Methods}

\section{Chamois sampling}

The respiratory and gastrointestinal systems of 28 adult Pyrenean chamois, 17 females (mean age 9.2 years, range $3-21$ ) and 11 males (mean age 7.5 years, range 3-12) from the Freser-Setcases National Game Reserve (Catalan Pyrenees, northeast Spain) were collected. Animals were hunter-harvested from October to December in 2012 and 2013, coinciding with the rut and a period of poor food quality [16]. Once the animal was dead, heart puncture was performed to obtain blood samples, which were allowed to clot at room temperature and centrifuged at $1800 \mathrm{G}$. Serum was transported at $4{ }^{\circ} \mathrm{C}$ and frozen at $-80{ }^{\circ} \mathrm{C}$ until analysis. Abdominal and thoracic viscera were extracted and kidneys were stored with their fat. The organs were placed in labelled plastic bags and transported in a refrigerated box at $4{ }^{\circ} \mathrm{C}$ to the laboratory, where the material was stored at $-20{ }^{\circ} \mathrm{C}$ until parasitological examination. Kidney fat reserves were used as a proxy for body condition in sampled chamois [11].

\section{Parasitological data}

Adult nematodes were recovered from the lungs, preserved in $70 \%$ ethanol and later mounted in lactophenol for identification [17]. Lung larvae were obtained using the Baermann method on $30 \mathrm{~g}$ of lung parenchyma. Liquid was collected and centrifuged $(800 \times g)$. Larvae were identified under a microscope using a Favatti chamber [18].

\section{Biochemical analysis}

PON-1 activity was determined using p-nitrophenyl acetate as a substrate [19]. Serum paraoxonase (PON1) is an enzyme able to neutralise lipid-peroxides (oxidised lipids) that cause inflammation among other organic and inorganic substrates. The TAC (ability of antioxidants to clear harmful free radicals) was measured using a method based on 2.2'-azinobis-(3-ethylbenzothiazoline6-sulfonate) decolorization by antioxidants [20]. TAC considers the cumulative action of all the antioxidants present in body fluids. Thus, a low TAC can be indicative of oxidative stress or increased susceptibility to oxidative damage. GPX was measured according to a previously described method [21]. The main biological role of GPX is to reduce lipid hydroperoxides to their corresponding alcohols and free hydrogen peroxide to water. All three analyses were measured in serum with an automated biochemistry analyser (Olympus AU600; Olympus Europe GmbH, Hamburg, Germany). IGF-1 is a hormone that plays an important role in the growth of juveniles and anabolism in adults and it was analysed with an automated solid-phase, enzyme-labelled chemiluminescent immunometric assay (Immulite System, Siemens Health Diagnostics, Deerfield, IL, USA). All analyses showed inter- and intra-assay coefficients of variation below $15 \%$, and recovery ranged between 80 and $120 \%$ in all cases.

\section{Statistical analyses}

The lung nematode load obtained in the present study was analysed in combination with the data on gastrointestinal parasite load recorded for the same individuals and previously published by Martínez-Guijosa et al. [3]. 
The partial least squares (PLS) regression method was used to assess the relationships between parasitism and health in female and male chamois. As a response variable, we defined a "Cost of infection block", which included markers of oxidative stress (GPX, PON1), antioxidant capacity (TAC), and the nutritional status of individuals (IGF-1 and KFs). The endoparasite infection block (set of predictor variables) was represented by the intensity of each parasite species (number of parasite individuals of each species), the total intensity (including all species) and the species richness of lung and gastrointestinal parasites. The PLS regression is suitable when sample size is low relative to the number of variables and when collinearities occur among predictor variables [22]. The loads, weights (i.e. relative contribution of each variable to the derived factors), and cross-correlations (e.g. the correlation between each $\mathrm{X}$ variable and the whole $\mathrm{Y}$ component, i.e. PLS' scores, and vice versa) were also estimated. Finally, the Stone-Geisser test $\left(\mathrm{Q}^{2}\right)$ at a threshold of 0.0975 was used to assess the fit of the PLS model. The $Q^{2}$ statistic can be viewed as a jackknife version of the $\mathrm{R}^{2}$ statistic. Analyses were performed with the "plspm" package [23] in R, version 3.3.2 [24].

\section{Results}

Similar to gastrointestinal parasites [3], prevalence and intensity of lung nematodes and their larvae were higher in males than in females (Table 1). Regarding the health indicators, however, only the PON1 concentration was significantly different between sexes after a Bonferroni correction $\left(t_{(24.8)}=5.03, P\right.$-value $<0.001$, Table 2$)$.

After a preliminary analysis, we decided to exclude the IGF-1 and TAC from the Y block due to their low contribution $(<2 \%)$ to the health block. Along the same lines, predictor variables with little or no influence on the X's component (e.g. burdens of specific parasite species, such as cestodes or Cooperia oncophora) were also discarded to simplify our PLS model. In female chamois, the health block showed no correlation with parasite infection $(\mathrm{Q} 2=-0.57)$. In contrast, the male group analysis revealed a marked correlation (5.7 times greater than females; $\mathrm{Q} 2=0.22$ ), where $57.1 \%$ of the observed variability of chamois health status was accounted for by parasite infections. As a result, gastrointestinal helminth richness, respiratory nematode richness and total gastrointestinal intensity, in decreasing order of importance, had the greatest negative effect on the $\mathrm{Y}$ block (Table 3, Fig. 1). In the case of females, only $1.9 \%$ of the health status was related to parasite infections.

\section{Discussion}

Chamois with low parasitic intensities and richness showed higher PON1 and GPX concentrations (for a given TAC concentration) and were in better body condition than their

Table 1 Prevalence at 95\% confidence interval $(95 \% \mathrm{Cl}$ ), mean and median intensity and its range (min-max) of adult and larval respiratory helminths in Pyrenean chamois (17 females and 11 males) from the Freser-Setcases National Game Reserve, northeast Spain

\begin{tabular}{|c|c|c|c|c|c|c|c|c|}
\hline & \multicolumn{2}{|c|}{$\begin{array}{l}\text { Prevalence (\%) } \\
(95 \% \mathrm{Cl})\end{array}$} & \multicolumn{2}{|c|}{ Mean intensity } & \multicolumn{2}{|c|}{ Median intensity } & \multicolumn{2}{|c|}{ Intensity range } \\
\hline & Male & Female & Male & Female & Male & Female & Male & Female \\
\hline \multicolumn{9}{|l|}{ Adult parasites } \\
\hline Protostrongylus rufescens boevi & $\begin{array}{l}9.1 \\
(0-26.1)\end{array}$ & $\begin{array}{l}0 \\
(0-0)\end{array}$ & 3 & 0 & 0 & 0 & $0-3$ & 0 \\
\hline Protostrongylus rufescens rufescens & $\begin{array}{l}90.9 \\
(73.9-100)\end{array}$ & $\begin{array}{l}43.8 \\
(19.4-68.1)\end{array}$ & 62.7 & 2 & 12 & 0 & $0-241$ & $0-5$ \\
\hline Protostrongylus rupicaprae & $\begin{array}{l}90.9 \\
(73.9-100)\end{array}$ & $\begin{array}{l}62.5 \\
(38.8-86.2)\end{array}$ & 89.3 & 4.1 & 36 & 2 & $0-343$ & $0-10$ \\
\hline Spiculocaulus austriacus & $\begin{array}{l}100 \\
(100-100)\end{array}$ & $\begin{array}{l}31.3 \\
(8.5-54.0)\end{array}$ & 52.5 & 2.4 & 17 & 0 & $1-311$ & $0-4$ \\
\hline Total & $\begin{array}{l}100 \\
(100-100)\end{array}$ & $\begin{array}{l}81.3 \\
(62.1-100)\end{array}$ & 191 & 5.2 & 70 & 4 & $1-845$ & $0-12$ \\
\hline \multicolumn{9}{|l|}{ Larval parasites } \\
\hline Muellerius spp. & $\begin{array}{l}63.6 \\
(35.2-92.1)\end{array}$ & $\begin{array}{l}56.3 \\
(31.9-80.6)\end{array}$ & 303.8 & 70.4 & 4 & 4 & $0-1669$ & $0-241$ \\
\hline Neostrongylus linearis & $\begin{array}{l}100 \\
(100-100)\end{array}$ & $\begin{array}{l}75.0 \\
(53.8-96.2)\end{array}$ & 128 & 165.9 & 23 & 13 & $1-594$ & 0-701 \\
\hline Protostrongylus spp. & $\begin{array}{l}100 \\
(100-100)\end{array}$ & $\begin{array}{l}68.8 \\
(46.0-91.5)\end{array}$ & 1256.4 & 126.8 & 107 & 7 & $1-10,609$ & $0-650$ \\
\hline Total & $\begin{array}{l}100 \\
(100-100)\end{array}$ & $\begin{array}{l}100 \\
(100-100)\end{array}$ & 1577.7 & 157.7 & 129 & 49 & $2-10,994$ & $1-1071$ \\
\hline
\end{tabular}


Table 2 Mean \pm standard deviation, minimum and maximum values of selected physiological indicators to assess the health status of Pyrenean chamois (17 females and 11 males) in Freser-Setcases National Game Reserve, northeast Spain

\begin{tabular}{lll}
\hline Health status indicators & Male & Female \\
\hline Oxidant status & & $14.68 \pm 3.91(5.28-19.33)$ \\
PON1 (units/l) & $8.57 \pm 2.39(5.81-12.63)$ & $549.75 \pm 300.95(192-1200)$ \\
GPX (units/mg) & $546.54 \pm 385.15(224-1532)$ & $0.35 \pm 0.18(0.02-0.68)$ \\
Antioxidant status & $0.29 \pm 0.13(0.11-0.48)$ & $60.36 \pm 28.69(13.71-137.01)$ \\
TAC (mmol/l) & & $35.36 \pm 6.08(25.30-50.91)$ \\
Nutritional status & $71.91 \pm 53.26(17.11-211.01)$ & $61.65 \pm 18.35(34.55-90.52)$ \\
IGF-1 (ng/ml) & $39.01 \pm 5.61(28.52-46.51)$ & \\
Kidney weight (g) & $53.25 \pm 12.53(33.55-76.80)$ &
\end{tabular}

Abbreviations: PON1 serum paraoxonase 1; GPX glutathione peroxidase; TAC total antioxidant capacity; IGF-1 insulin-like growth factor 1

more parasitized counterparts. On the other hand and line with previous work [25], we found that multiple infections have a greater physiological cost than single-parasite infections. However, interpreting sex differences in the physiological cost of parasitism is more challenging for several reasons, the first being the fact that females were by far less parasitized than males.

In males, the poor body condition and reduced antioxidant defences in highly parasitized individual chamois suggest an overload in the host's capacity to overcome the infestation. Lower parasite burdens and richness show higher levels of antioxidant biomarkers (PON1 and GPX), resulting from up-regulation of endogenous antioxidants or mobilization of exogenous antioxidants, along with better body condition. Nonetheless, both sexes have similar lifespan, suggesting that male chamois employ compensatory mechanisms to deal with the extracost of parasite infections during the rut season. Females, however, do not seem to need these physiological compensations at this time of the year.

Many parasite infections in wildlife tend to be relatively benign, remaining subclinical until the host experiences additional stress [26]. Thus, many of the pathogenic mechanisms would be driven by the host's own immune system, which is also energetically demanding. In these cases, when the cost of maintaining resistance outweighs the benefits, it has been postulated that tolerance is preferred [27]. This strategy, based on reducing the fitness or health cost of an increasing parasite burden, has been described as naturally selected in ungulates. It is especially advantageous when resources must be allocated in confronting more severe threats [28]. Hence, male chamois

Table 3 Predictor weights of the most parsimonious Partial Least Squares (PLS) regression model explaining the physiological cost of parasitism in male chamois from the Freser-Setcases National Game Reserve, northeast Spain

\begin{tabular}{llllll}
\hline PLS blocks & Predictor variables & Loads & Weights & Percent & Cross-correlation \\
\hline X Gl richness & -0.41 & -0.54 & 29.25 & -0.99 \\
& LN richness & -0.37 & -0.46 & 21.41 & -0.85 \\
Total GI intensity & -0.37 & -0.39 & 15.04 & -0.71 \\
Nematodirus spp. & -0.31 & -0.32 & -0.51 & -0.49 & -0.47 \\
Marshallagia spp. & -0.19 & -0.25 & 6.12 & -0.45 \\
Total LN intensity & -0.27 & -0.25 & 5.48 & -0.43 \\
P. r. rufescens & -0.28 & -0.23 & 5.41 & -0.43 \\
S. austriacus & -0.27 & -0.23 & 0.29 & -0.10 \\
LN larval richness & -0.08 & -0.05 & 81.81 & 0.83 \\
PON1 & 0.44 & - & 88.34 & 0.72 \\
GPX & 0.37 & - & 77.98 & 0.71 \\
\hline
\end{tabular}

Predictor variables explaining more than $10 \%$ in the response block are shown in bold type. In the PLS Y's block the $\mathrm{Y} U$ correlation has been shown as a proxy for variable importance

Abbreviations: Gl richness, number of gastrointestinal helminth species; LN richness, number of lung nematode species; Total Gl intensity, number of adult nematodes in the gastrointestinal tract; Total LN intensity, number of adult nematodes in the lung; LN larval richness, number of larvae nematode species in the lung 


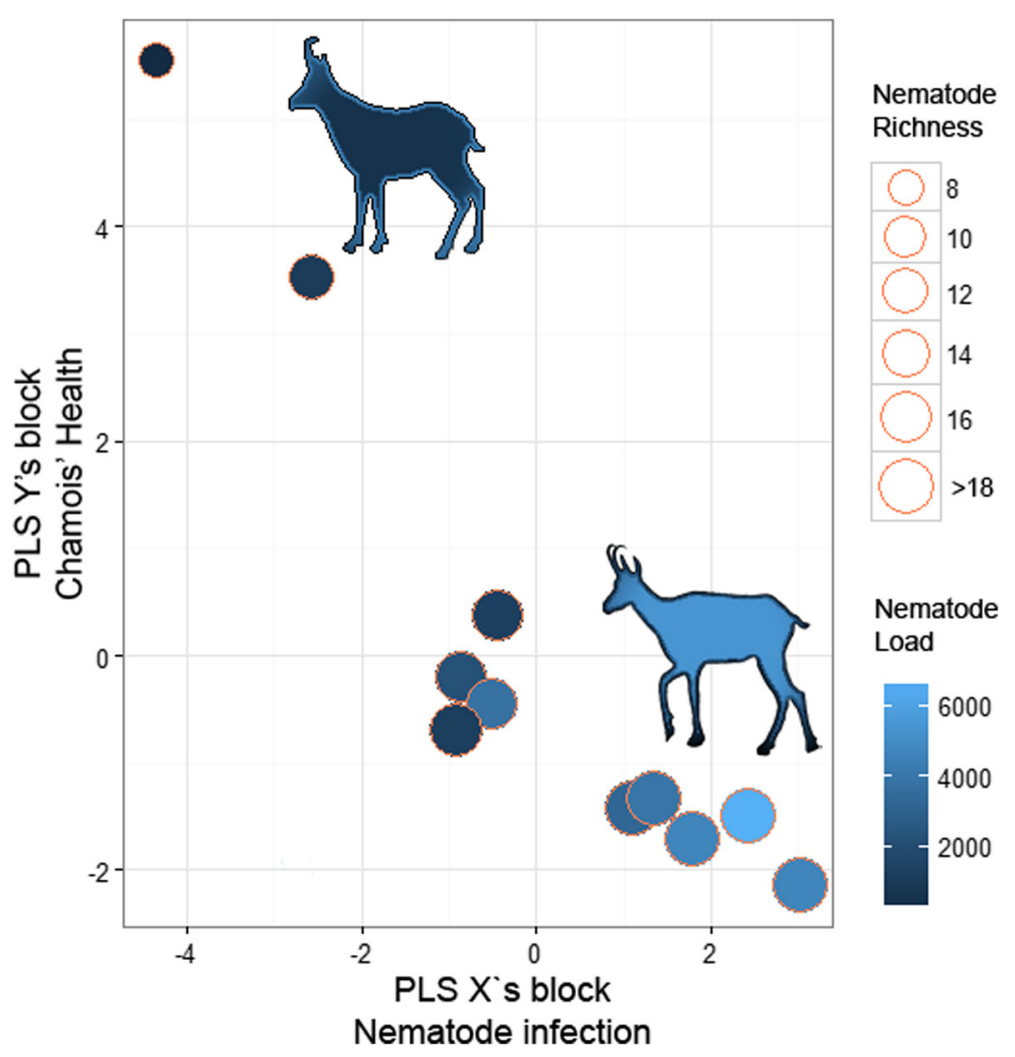

Fig. 1 Bubble chart showing the relationships between PLS X's component, representing the gut and lung nematode community, and the PLS Y's component representing the health status in eleven male chamois from the Catalan Pyrenees, northeast Spain. The nematode community block was mainly represented by gastrointestinal and lung nematode richness and by gastrointestinal nematode intensity of infection, whereas the health block was represented by glutathione peroxidase (GPX) and paraoxonase-1 (PON1) concentrations, and by body condition. The initial set of variables representing each block is provided in Table 3. Bubble size indicates total (gut and lung) nematode richness ranging from 0 to 19 nematode species. Bubble colour indicates nematode burden. Chamois silhouettes represent a male chamois with low parasite load and hence in good condition and with a good antioxidant response (top left) and a highly parasitized male chamois in poorer condition and lower antioxidant response (bottom right)

may need to increase their tolerance (through raising PON1 and GPX concentrations) during the rut period, when parasite burdens increase due to the immunosuppressive influence of their reduced feeding coupled with the high testosterone levels. Males could then invest their resources in eliminating parasites or reducing their burdens, and repairing tissue lesions when the rut period is finished, their appetite returns to normal and their immune system is relieved of the aforementioned challenges. The increases in body mass that males show in summer [29] may also contribute to compensate a further cost of parasitism during the rut.

The lack of any correlation between health status and parasite infections in females could be explained by their low parasite burden at this time of year (Table 1 and [3]). Some evidence supports this hypothesis. Hypophagia displayed by males during the rutting period, especially in territorial individuals [15], would diminish the probability of introducing new infective (L3) larvae from contaminated grass. Additionally, L3 larvae of most gastrointestinal nematode species in non-naïve Caprinae take a minimum of approximately 3-4 weeks to reach adulthood [30] and, if ingested in late summer/early fall, will most likely be hypobiotic for months unless reawakened by a stressor [31]. Hence, both hypophagia and the long time required by larvae to reach adulthood, may suggest that the higher parasite richness and burden of males at that period is not only due to high testosterone concentrations, but the consequence of previously acquired infections. In fact, only one female displayed a parasite burden (2209 nematodes) close to the mean value of males (2797 nematodes). Thus, this work demonstrates that being a male has an extra physiological cost due to sex-biased parasitism that is most likely exacerbated in the autumn. This situation results from the combination of multiple parasite infections and a debilitated immune system due to malnourishment and high levels of testosterone during rut. We hypothesized that the correlation among health parameters and parasite burden is most likely initiated when tolerance to parasite infection is exceeded. 
Other hypotheses should also be considered. Individual male chamois differ extensively in their energy investment in rutting activities in specific years [32]. Hence, many individuals may not be exposed to some of the detrimental effects, therefore, reducing the overall differences in mortality between males and females. Moreover, females could have their own health depletion period, such as during lactation or pregnancy, differentiated in time from the male depletion periods. This could lead males and females to be under similar stress pressures over a year-long period.

\section{Conclusions}

Despite the moderate sample size, this study showed a sex-biased physiological cost of multiple infections by gastrointestinal and respiratory nematode parasites in a wild mammal. Interestingly, this extra-cost is mainly related to the number of different parasite species and, to a lesser extent, with the intensity of infection. The similar lifespan of females and males of this mammal suggest that the latter can deal with this cost of infection, at least during the rutting period. Further research should investigate whether this sex-biased cost of infection persists over the year and whether females suffer from these consequences of parasite infection at other times of year. On the other hand, individual-based information will be required to better understand the mechanisms driving the sex-biased parasitism, its physiological cost, and in turn, survival probabilities of this mountain ungulate.

\section{Abbreviations \\ GPX: Glutathione peroxidase; IGF1: Insulin-like growth factor; KFs: Kidney fat stores; OS: Oxidative stress; PLS: Partial least squares; PON1: Paraoxonase 1; $Q^{2}$ : Stone-Geisser test; $R^{2}: R$ squared or coefficient of determination; TAC: Total antioxidant capacity}

\section{Acknowledgements}

We would like to thank Mr. Jordi Xifra of the National Game Reserve of Freser-Setcases and all the game keepers from the Cos d'Agents Rurals for their support during the Pyrenean chamois sampling.

\section{Funding}

JMG holds a FPI pre-doctoral scholarship (BES-2015-072206), funded by the Spanish Ministry of Economy and Competitiveness. ES was supported by the postdoctoral programme (SFRH/BPD/96637/2013) of the Fundação para a Ciência ea Tecnologia, Portugal. We would like to thank the University of Aveiro (Department of Biology) and FCT/MEC for the financial support to CESAM RU (UID/AMB/50017) through national funds and, where applicable, co-financed by the FEDER, within the PT2020 Partnership Agreement. This study was partly funded by the scientific programme "Approche Intégrée de la Démographie des Populations d'Isard" sponsored by the ONCFS and the SEFaS.

\section{Availability of data and materials}

Data from the study are available upon request from the corresponding author (ES).

\section{Authors' contributions}

Designed the study: AOG, CMC, JRLO, LR and ES. Performed the collection of respiratory systems: JRLO, GM, XFA, ACC and DG. Necropsies were performed by: AOG, CMC, MRRY, RV, DG and JMG. Biochemical analysis: AT. Analysed the data: $\mathrm{AOG}$ and ES. Wrote the paper: AOG, CMC, SL, MG and ES. All authors read and approved the final manuscript.

\section{Competing interests}

The authors declare that they have no competing interests.

\section{Consent for publication}

Not applicable.

\section{Ethics approval}

No approval was needed from any Ethics committee since the animals used in the present study were not sacrificed for research purposes. The harvested chamois included in the present study have been legally hunted (shot) by authorized gamekeepers within the framework of an annual hunting plan approved by the Departament d'Agricultura, Ramaderia, Pesca, Alimentacio'i Medi Natural - Generalitat de Catalunya, Spain.

\section{Author details}

'Servei d'Ecopatologia de Fauna Salvatge (SEFaS), Wildlife Health Service, Departament de Medicina i Cirurgia Animal, Universitat Autònoma de Barcelona, E-08193 Bellaterra, Spain. ${ }^{2}$ Departamento de Sanidad Animal, Facultad de Veterinaria, Campus de Excelencia Internacional Regional "Campus Mare Nostrum", Universidad de Murcia, E-30100 Murcia, Spain. 3Departamento de Medicina y Cirugía Animal, Facultad de Veterinaria, Campus de Excelencia Internacional Regional "Campus Mare Nostrum", Universidad de Murcia, Campus de Espinardo, E-30100, Espinardo, Murcia, Spain. Instituto de Investigación en Recursos Cinegéticos IREC (CSIC-UCLM-JCCM), E-13071 Ciudad Real, Spain. ${ }^{5}$ Unité Faune de Montagne, Office National de la Chasse et de la Faune Sauvage (ONCFS), 34990 Juvignac, France. ${ }^{6}$ Dipartimento di Scienze Veterinarie, Università degli Studi di Torino, Grugliasco, 10095 Torino, Italy. ${ }^{7}$ Departamento de Biologia \& CESAM, Universidade de Aveiro, 3810-193 Aveiro, Portugal.

Received: 9 December 2016 Accepted: 23 February 2017

Published online: 21 April 2017

\section{References}

1. Zuk M, McKean KA. Sex differences in parasite infections: patterns and processes. Int J Parasitol. 1996;26:1009-24.

2. Poulin R. Evolutionary Ecology of Parasites. Princeton: Princeton University Press; 2007

3. Martínez-Guijosa J, Martínez-Carrasco C, López-Olvera JR, Fernández-Aguilar X, Colom-Cadena A, Cabezón O, et al. Male-biased gastrointestinal parasitism in a nearly monomorphic mountain ungulate. Parasit Vectors. 2015:8:165.

4. Guerra-Silveira F, Abad-Franch F. Sex bias in infectious disease epidemiology: patterns and processes. PLoS One. 2013;8:e62390.

5. Connors VA, Nickol BB. Effects of Plagiorhynchus cylindraceus (Acanthocephala) on the energy metabolism of adult starlings, Sturnus vulgaris. Parasitology. 1991;103:395-402.

6. Krasnov BR, Bordes F, Khokhlova IS, Morand S. Gender-biased parasitism in small mammals: patterns, mechanisms, consequences. Mammalia. 2012;76:1-13.

7. Klein SL. Hormonal and immunological mechanisms mediating sex differences in parasite infection. Parasite Immunol. 2004;26:247-64.

8. Folstad I, Karter AK. Parasites, bright males and the immunocompetence handicap. Am Nat. 1992;139:603-22.

9. Promislow DE. Costs of sexual selection in natural populations of mammals. Proc Roy Soc B Biol Sci. 1992;247:203-10.

10. Gonzalez G, Crampe J-P. Mortality patterns in a protected population of isards (Rupicapra pyrenaica). Can J Zool. 2001;79:2072-9.

11. Serrano E, Alpizar-Jara R, Morellet N, Hewison A. A half a century of measuring ungulate body condition using indices: is it time for a change? Eur J Wildl Res. 2008;54:675-80.

12. Valko M, Leibfritz D, Moncol J, Cronin MTD, Mazur M, Telser J. Free radicals and antioxidants in normal physiological functions and human disease. Int J Biochem Cell Biol. 2007;39:44-84

13. Lykkesfeldt J, Svendsen O. Oxidants and antioxidants in disease: Oxidative stress in farm animals. Vet J. 2007:173:502-11.

14. Beaulieu M, Costantini D. Biomarkers of oxidative status: missing tools in conservation physiology. Conserv Physiol. 2014;2:cou014. doi:10.1093/ conphys/cou014.

15. Corlatti L, Bassano B. Contrasting alternative hypotheses to explain rutinduced hypophagia in territorial male chamois. Ethology. 2014;120:32-41. 
16. Villamuelas M, Fernández N, Albanell E, Gálvez-Cerón A, Bertolomé J, Mentaberre G, et al. The Enhanced Vegetation Index (EVI) as a proxy for diet quality and composition in a mountain ungulate. Ecol Indic. 2016;61:658-66.

17. Gassó D, Rossi L, Mentaberre G, Casas E, Velarde E, Nosal P, et al. An identification key for the five most common species of Metastrongylus. Parasitol Res. 2014;113:3495-500.

18. Demolin D. Identification des endoparasites du chamois (Rupicapra rupicapra L.) en France. Thèse présentée à I'Université Claude-Bernard de Lyon (Médecine-Pharmacie). École Nationale Vétérinaire de Lyon; 1984.

19. Tvarijonaviciute A, Tecles F, Caldin M, Tasca S, Cerón J. Validation of spectrophotometric assays for serum paraoxonase type-1 measurement in dogs. Am J Vet Res. 2012;73:34-41.

20. Erel O. A novel automated method to measure total antioxidant response against potent free radical reactions. Clin Biochem. 2004;37:112-9.

21. Paglia DE, Valentine WN. Studies on the quantitative and qualitative characterization of erythrocyte glutathione peroxidase. J Lab Clin Med. 1967; 70:158-69.

22. Carrascal LM, Galván I, Gordo O. Partial least squares regression as an alternative to current regression methods used in ecology. Oikos. 2009;118:681-90.

23. Sánchez G, Trinchera L, Russolillo G. plspm: Tools for Partial Least Squares Path Modeling (PLS-PM). R package version 0.4.7. 2015. http://CRAN.Rproject.org/package=plspm. Accessed Jan 12017.

24. R Core Team. R. A language and environment for statistical computing. $R$ Foundation for Statistical Computing, Vienna, Austria. 2017; URL https:// www.R-project.org/. Accessed Jan 12017.

25. Serrano E, Millán J. What is the price of neglecting parasite groups when assessing the cost of co-infection? Epidemiol Infect. 2014;142:1533-40.

26. Mayer M, Brown GP, Zimmermann B, Shine R. High infection intensities, but negligible fitness costs, suggest tolerance of gastrointestinal nematodes in a tropical snake. Aust J Ecol. 2015;40:683-92.

27. López Pascua L, Hall AR, Best A, Morgan AD, Boots M, Buckling A. Higher resources decrease fluctuating selection during host-parasite co-evolution. Ecol Lett. 2014;17:1380-8.

28. Hayward AD, Nussey DH, Wilson AJ, Berenos C, Pilkington JG, Watt KA, et al. Natural selection on individual variation in tolerance of gastrointestinal nematode infection. PLoS Biol. 2014;12:e1001917.

29. Garel M, Loison A, Jullien J-M, Dubray D, Maillard D, Gaillard J-M. Sexspecific growth in Alpine chamois. J Mammal. 2009;90:954-60.

30. Gómez-Muñoz MT, Cuquerella M, Gómez-Iglesias LA, Méndez S, FernándezPérez FJ, de la Fuente C, et al. Serum antibody response of Castellana sheep to Haemonchus contortus infection and challenge: relationship to abomasal worm burdens. Vet Parasitol. 1999;81:281-93.

31. Sargison ND, Wilson DJ, Bartley DJ, Penny CD, Jackson F. Haemonchosis and teladorsagiosis in a Scottish sheep flock putatively associated with the overwintering of hypobiotic fourth stage larvae. Vet Parasitol. 2007:47:326-31.

32. Corlatti L, Caroli M, Pietrocini L, Lovari S. Rutting behavior of territorial and non-territorial male chamois: Is there a home range advantage? Behav Process. 2013;92:118-24.

\section{Submit your next manuscript to BioMed Central and we will help you at every step:}

- We accept pre-submission inquiries

- Our selector tool helps you to find the most relevant journal

- We provide round the clock customer support

- Convenient online submission

- Thorough peer review

- Inclusion in PubMed and all major indexing services

- Maximum visibility for your research

Submit your manuscript at www.biomedcentral.com/submit

CBiomed Central 